\title{
Dreiste Patienten, dreiste Einweiser - wann darf ich den Transport verweigern?
}

Andreas Staufer

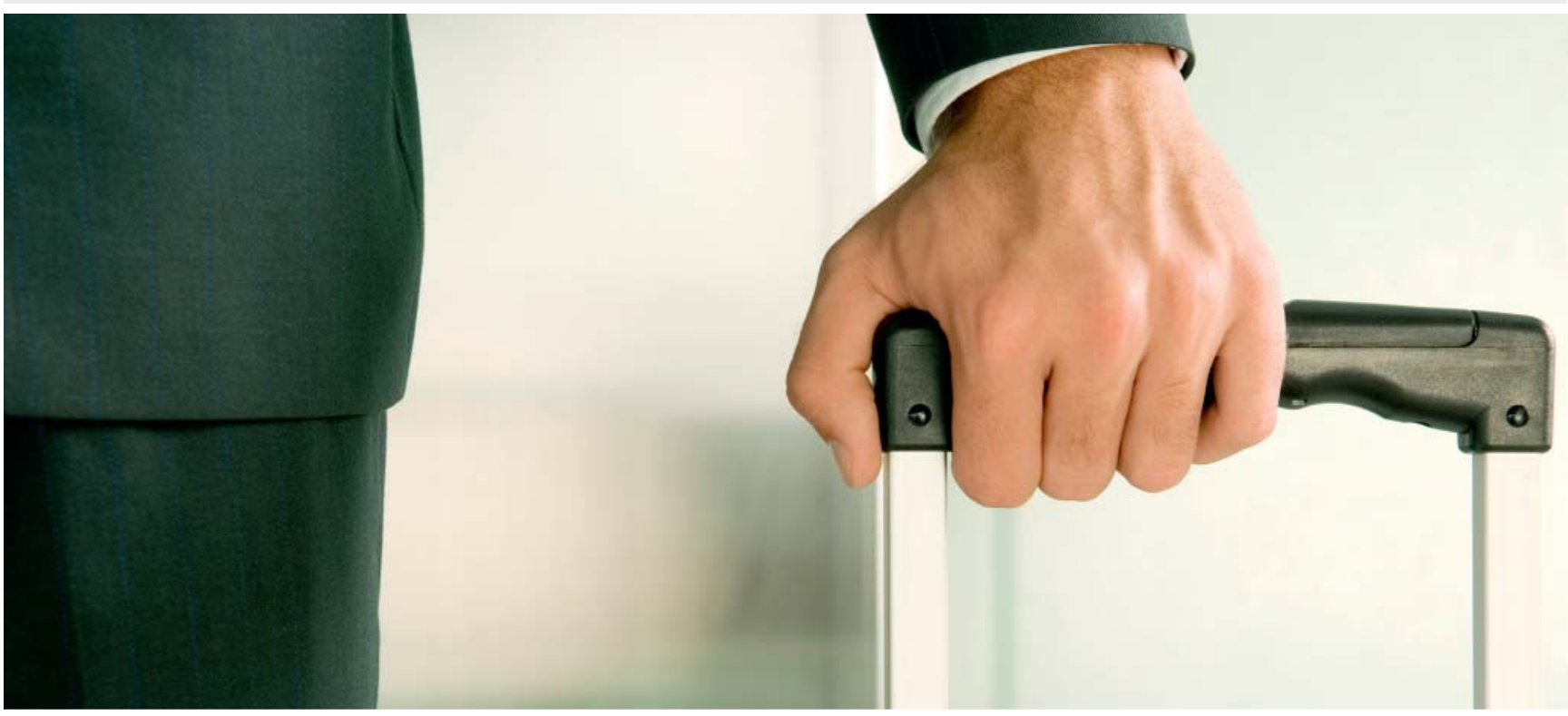

Quelle: flairimages/AdobeStock

Die Anfahrt erfolgt mit Blaulicht und Martinshorn, doch der Patient wartet bereits mit gepackten Koffern und fordert den Transport in eine ihm genehme Klinik. Transportindikation? Keine ersichtlich. Schlimmer noch: Der Patient zeigt sich gegenüber dem Rettungsdienst äußerst aggressiv; die Mitarbeiter fühlen sich gefährdet. Wie dürfen oder müssen sie im Rettungsdienst reagieren?

\section{Nicht immer ist der Patientenwille entscheidend}

Oft wird nur die Transportverweigerung durch den Patienten thematisiert. Seltener erörtert wird die Situation, dass der Patient transportiert werden will oder aufgrund der Vorgabe Dritter befördert werden soll - der Rettungsdienst dies allerdings nicht für notwendig erachtet oder sich den Transport nicht zutraut. Drei Fallbeispiele sollen das verdeutlichen.

\section{Fallbeispiel 1}

Der Patient wartet bereits mit gepackten Koffern. Er ist für eine seit Langem geplante plastische Operation einbestellt. Der Arzt habe am Telefon darauf verwiesen, dass bei einem Notfalltransport kein Eigenanteil bei der Krankenkasse anfalle. Ein Taxi wolle er sich nicht leisten. Eine Verordnung liegt nicht vor.

\section{Fallbeispiel 2}

Der Rettungsdienst soll einen intensivpflichtigen Patienten aus einem Klinikum der Maximalversorgung verlegen. Der Transport ist nicht eilig. Dafür erweist sich der Patient als übergewichtig und zu schwer für die Transportliege. Die Besatzung kann die mitzuführenden Medizinprodukte der Klinik nicht sicher verlasten. Ein nicht qualifizierter Assistenzarzt soll den Transport begleiten.

\section{Fallbeispiel 3}

Nach einer Schlägerei bittet die Polizei den Rettungsdienst, den sich noch immer heftig wehrenden, aber gefesselten und mit Schnittwunden übersäten Patienten in eine Klinik zu fahren. Beim Nähern an die Trage tritt der Patient den Sanitäter heftig mit den verbundenen Füßen. Die Polizei ist vor Ort gebunden und kann den Transport nicht begleiten [3]. 


\section{Aufgaben des Rettungsdienstes}

Die Bundesländer regeln die Aufgaben ihrer Rettungsdienste aufgrund des Art. 70 Abs. 1 Grundgesetz in jeweils eigener Gesetzgebungskompetenz. Die Ausbildung der Notfallsanitäter als Berufsausbildung ist dagegen durch ein Bundesgesetz geregelt. Eine Übersicht der verschiedenen Landesrettungsdienstgesetze ist online verfügbar [1].

Allerdings definieren die Bundesländer die Aufgaben des Rettungsdienstes weitestgehend gleichlautend.

\section{Notfallrettung und Krankentransport}

Die Notfallrettung umfasst die notfallmedizinische Versorgung von Notfallpatienten am Notfallort und den Notfalltransport. Als Notfallpatienten werden meist Verletzte oder Kranke definiert, die sich in Lebensgefahr befinden oder bei denen schwere gesundheitliche Schäden zu befürchten sind, wenn sie nicht unverzüglich die erforderliche medizinische Versorgung erhalten. Sie sind unter fachgerechter medizinischer Betreuung in eine für die weitere Versorgung geeignete Einrichtung zu befördern.

Krankentransport ist der Transport von kranken, verletzten oder sonstigen hilfsbedürftigen Personen, die - ohne Notfallpatienten zu sein - während der Fahrt einer fachlichen medizinischen Betreuung durch nichtärztliches medizinisches Fachpersonal oder der besonderen Einrichtungen des Krankenkraftwagens bedürfen. So die gesetzliche Definition in $\S 2$ Abs. 2 Satz 1, Abs. 5 Satz 1 BayRDG.

\section{Beförderungspflicht}

Die mit der Notfallrettung und dem Krankentransport Beauftragten trifft meist eine gesetzliche Beförderungspflicht. Keine Beförderungspflicht besteht allerdings bei Transporten, die nicht der Notfallrettung oder dem Krankentransport zuzuordnen sind.

Nicht zum Krankentransport gehört die Beförderung kranker Personen, die während der Beförderung keiner fachlichen medizinischen Betreuung oder der besonderen Einrichtungen des Krankenkraftwagens bedürfen (Krankenfahrten). Gleiches gilt für Beförderungen, die nicht in die nächste für die weitere Versorgung geeignete und aufnahmebereite Einrichtung führen, die tatsächlich bzw. aus rechtlichen Gründen nicht durchgeführt werden dürfen, die für die Besatzung aus anderen Gründen unzumutbar sind oder die aus anderen Gründen von den Durchführenden nicht zu vertreten sind.

\section{Cave}

Bei Notfallpatienten darf die Beförderung nicht deshalb abgelehnt werden, weil ein rechtswirksamer Beförderungsvertrag nicht vorliegt oder die Entrichtung des Entgelts nicht gesichert ist.
Die Beförderungspflicht in der Notfallrettung ist also unabhängig von der Sicherstellung des Beförderungsentgelts.

Welche Fahrten die für die gesetzliche Krankenversicherung zugelassenen Ärzte verordnen dürfen, bestimmen das Fünfte Buch Sozialgesetzbuch (SGB V) sowie die vom Gemeinsamen Bundesausschuss (G-BA) erlassene Krankentransport-Richtlinie [2]. Auch bei privat und anderweitig versicherten Patienten ist eine medizinische Notwendigkeit der Fahrt meist Voraussetzung für die Kostenerstattung.

\section{Bewertung der Fallbeispiele}

Der Patient in Fallbeispiel 1 fällt mangels einer medizinischen Indikation nicht unter die gesetzliche Beförderungspflicht.

In Fallbeispiel 2 ist die Beförderung aufgrund der fehlenden Qualifikation sowie der fehlenden Ladungssicherheit nicht möglich; in diesem Fall würde die Durchführung der Beförderung auch gegen das Medizinprodukterecht sowie die in $\S 22$ der Straßenverkehrsordnung normierte Ladungssicherheit verstoßen.

Im dritten Fallbeispiel ist die Besatzung berechtigt, die Beförderung aufgrund der Selbstgefährdung abzulehnen.

\section{Behandlungspflicht}

Von der Beförderungspflicht zu unterscheiden ist die Pflicht zur Lageerkundung, zur Beurteilung des Gesundheitszustands des Patienten und zur Durchführung medizinischer Maßnahmen der Erstversorgung. Danach ist jedermann verpflichtet, anderen Menschen in Not zu helfen. Wer das nicht für nötig erachtet, obwohl dies erforderlich und ihm den Umständen nach zuzumuten ist, kann sich wegen unterlassener Hilfeleistung nach $\S 323 \mathrm{c}$ StGB strafbar machen.

Mitarbeiter im Rettungsdienst haben darüber hinausgehende Pflichten. Ihnen fällt gegenüber den Patienten eine Garantenstellung mit besonderen Obhutspflichten zu. Kommt es infolge des Unterlassens einer Maßnahme zu einer Schädigung des Patienten oder zu dessen Tod, können die Straftatbestände der Körperverletzung oder des Totschlags erfüllt sein.

Lässt der Rettungsdienst beispielsweise bei Minusgraden einen alkoholisierten Patienten ohne weitere Hilfe unbedacht im Kalten liegen und stirbt dieser, so kann nicht nur der Straftatbestand der unterlassenen Hilfeleistung erfüllt sein, sondern auch der der fahrlässigen Tötung.

Selbst bei unangenehmen, querulatorischen oder besserwisserischen Patienten bleibt daher im Rahmen des Vertretbaren und vom Patienten Geduldeten eine sorgfältige 
Beurteilung unerlässlich. Diese ist so sorgfältig zu dokumentieren, dass sie noch nach mehreren Jahren hinreichende Rückschlüsse auf den Vorgang und den Verlauf des Einsatzes erlaubt.

Eine Behandlungspflicht besteht allerdings nur, wenn eine Indikation dazu vorliegt und der Rettungsdienst nicht aus anderen Gründen von dieser Pflicht entbunden ist - etwa, weil er sich selbst in erhebliche Gefahr bringen würde.

\section{Bewertung der Fallbeispiele}

Im ersten Fallbeispiel ist keine Behandlungspflicht gegeben. Der Patient ist nicht in Not. Ebenso fehlt es an der Erforderlichkeit einer medizinischen Behandlung.

Im zweiten Fallbeispiel ist die Betreuung des Patienten im Klinikum sichergestellt. Zu einer Beförderung ist die Besatzung mangels Qualifikation und Ressourcen nicht verpflichtet.

Im dritten Fallbeispiel kann die Besatzung aufgrund des Verhaltens des Patienten ihre Behandlungspflicht nicht erfüllen. Weder zeigt der Patient durch sein Verhalten, dass er eine Behandlung wünscht, noch ist angesichts des aggressiven Verhaltens des Patienten dem Rettungsdienst die Behandlung zumutbar.

\section{Maßnahmen bei Ablehnung eines Transports}

Bevor eine Besatzung des Rettungsdienstes den Transport eines Patienten ablehnt, ist sie zu einer besonders sorgfältigen Feststellung und Erfassung der Lage und Beurteilung des Gesundheitszustands des Patienten unter Abwägung der Situation und der Möglichkeiten verpflichtet. Das gilt vor allem für augenscheinlich typische Fälle wie den eines alkoholisierten Patienten.

\section{Merke}

In zweifelhaften Fällen sollte die Besatzung den Patienten befördern oder einen Arzt zu Rate ziehen. Das kann bei fehlender Indikation und Dringlichkeit einer Beförderung auch ein ärztlicher Bereitschaftsdienst sein.

\section{Dokumentation der Maßnahmen}

Lehnt der Rettungsdienst die Beförderung ab, sollte er die getroffenen Feststellungen und Maßnahmen ausführlich dokumentieren. Gegebenenfalls hat er auf alternativ in Betracht kommende Leistungserbringer - wie Taxi und Mietwagen - hinzuweisen.

Bei hilflosen, aber nicht erkrankten oder verletzten Personen ist anderweitig adäquate Hilfe sicherzustellen, beispielsweise die Überwachung durch Angehörige, die Hinzuziehung anderer zuständiger Behörden wie des
Jugendamts oder der Polizei. Das Gleiche gilt entsprechend bei möglicher Selbstgefährdung.

\section{Zweifel an einer ärztlichen Verordnung}

Hat die Besatzung Zweifel an der Richtigkeit der ärztlichen Verordnung eines Krankentransports, empfiehlt sich eine Rücksprache mit dem ausstellenden Arzt. Ansonsten sollte sie den Transport durchführen und bestenfalls auch den Versuch der Rücksprache mit dem Arzt dokumentieren.

\section{Hinweis auf Kostentragung}

Befördert man den Patienten einvernehmlich auf dessen ausdrücklichen Willen, ist der einsichtsfähige Patient darauf hinzuweisen, dass er bei fehlender ärztlicher Verordnung die Kosten möglicherweise selbst zu tragen hat. Die wirtschaftliche Aufklärung des Patienten ist zu dokumentieren. Bestenfalls bestätigt der Patient die Aufklärung durch seine Unterschrift auf einem von dem Durchführenden herausgegebenen und rechtlich geprüften Formular. Übrigens kann auch bei vorsätzlich oder grob fahrlässig verursachten Fehlfahrten der Veranlasser verpflichtet sein, die Kosten des Einsatzes zu übernehmen.

\section{Fehlende Qualifikation und Ressourcen}

Fehlt es an der notwendigen Qualifikation bzw. den erforderlichen Ressourcen für die Fahrt, sind die bestehenden Bedenken gegenüber dem Auftraggeber deutlich zu kommunizieren und zu begründen. Auf alternative Beförderungswege ist hinzuweisen. Gegebenenfalls ist mit dem eigenen Dienstvorgesetzten oder der Leitstelle Rücksprache zu halten. Keinesfalls sollte die Besatzung eine Aufgabe übernehmen, die sie erkennbar nicht beherrscht andernfalls droht ihr zivil- oder strafrechtliche Verfolgung.

\section{KERNAUSSAGEN}

- Der Rettungsdienst ist nicht verpflichtet, jeden Fahrgast zu befördern. Eine Beförderungspflicht trifft ihn nur bei erforderlichen Leistungen der Notfallrettung und des Krankentransports.

- Maßnahmen sind sorgfältig zu dokumentieren. Im Zweifel ist der Patient zu transportieren.

- Soweit eine erhebliche Eigengefährdung vorliegt, besteht keine Verpflichtung zur Behandlung oder Beförderung. Kann eine Besatzung den Patienten mangels Qualifikation oder Ressourcen ersichtlich nicht befördern, muss sie den Transport ablehnen. Bedenken sind rechtzeitig mitzuteilen. Alternative Möglichkeiten sind zu prüfen.

- Da jeder Einzelfall individuell zu beurteilen ist, empfiehlt sich für Arbeitgeber, die Mitarbeiter durch eindeutige interne Vorgaben, Checklisten und Schulungen zu diesen Themen zu unterstützen und zu sensibilisieren. 
Die Autoren geben an, dass kein Interessenkonflikt besteht.

Autorinnen/Autoren

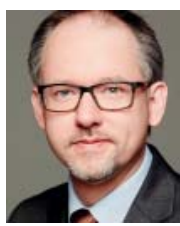

\section{Dr. iur. Andreas Staufer}

Fachanwalt für Medizinrecht und Fachanwalt für Informationstechnologierecht, spezialisiert auf das Rettungsdienstrecht sowie den Datenschutz im Gesundheitswesen. Lehrbeauftragter an der LMU München. Seit über 15 Jahren Rettungssanitäter in der Münchner Notfallrettung.

\section{Korrespondenzadresse}

Dr. iur. Andreas Staufer

FASP Finck Sigl \& Partner, Rechtsanwälte Steuerberater mbB Nußbaumstraße 12

80336 München

info@staufer.de

www.staufer.de
Literatur

[1] Gesetzessammlung Rettungsdienst. Im Internet: http://www. rettungsdienstgesetze.de; Stand: 11.03.2018

[2] Richtlinie über die Verordnung von Krankenfahrten, Krankentransportleistungen und Rettungsfahrten nach § 92 Absatz 1 Satz 2 Nummer 12 SGB V vom 22.01.2004. Bundesanzeiger Nr. 18 (Seite 1342) vom 28.01.2004 in seiner letzten Änderung vom 21.09.2017

[3] Beispiel nach Hochstein, Rechtliche Aspekte der Transportverweigerung, Vortrag vom 11.05.2015. Online abrufbar unter https://thomas-hochstein.de/download/rechtlicheaspekte-transportverweigerung.pdf zuletzt abgerufen am 30.07.2018

Bibliografie

DOI https://doi.org/10.1055/a-0495-1582

retten 2018; 7: 242-245

(c) Georg Thieme Verlag KG, Stuttgart · New York ISSN 2193-2387 